\title{
ASYMPTOTIC BEHAVIOR OF MEROMORPHIC FUNCTIONS WITH EXTREMAL DEFICIENCIES
}

\author{
BY ALBERT EDREI AND ALLEN WEITSMAN ${ }^{1}$
}

Communicated by Maurice Heins, September 18, 1967

Let $f(z)$ be a meromorphic function; it is assumed that the reader is familiar with the following symbols of frequent use in Nevanlinna's theory

$$
n(r, f), \quad N(r, f), \quad T(r, f), \quad \delta(\tau, f) .
$$

The lower order $\mu$ and the order $\lambda$ of $f(z)$ are defined by the familiar relations

$$
\liminf _{r \rightarrow \infty} \frac{\log T(r, f)}{\log r}=\mu, \quad \limsup _{r \rightarrow \infty} \frac{\log T(r, f)}{\log r}=\lambda .
$$

In addition to these classical concepts, we consider the total deficiency $\Delta(f)$ of the function $f$

$$
\Delta(f)=\sum_{\tau} \delta(\tau, f)
$$

where the summation is to be extended to all the values $\tau$, finite or $\infty$, such that

$$
\delta(\tau, f)>0 .
$$

The number of deficient values of $f$, that is the number of distinct values of $\tau$ for which (1) holds, will be denoted by $\nu(f)(\leqq+\infty)$.

The investigation presented here leads to the proof of

TheOREM A. Let $f(z)$ be a meromorphic function of lower order $\mu$ :

$$
\frac{1}{2}<\mu<1,
$$

and let the poles of $f(z)$ have maximum deficiency $(\delta(\infty, f)=1)$.

Then

$$
\Delta(f) \leqq 2-\sin \pi \mu .
$$

Moreover, if equality holds in (3), then

$$
\nu(f)=2 \text {. }
$$

1 The authors gratefully acknowledge support by the National Science Foundation under grants GP-5019 and GP-7507. 
Theorem A remains valid if, in (2) and (3), the lower order $\mu$ is replaced by the order $\lambda$. If we perform this substitution, the assertion $\lambda=\mu$ follows from the assumption $\Delta(f)=2-\sin \pi \lambda$.

With $\mu$ replaced by $\lambda$, the inequality (3) is known [4, Corollary 1.3 , p. 235]; in its present form, which does not exclude functions of infinite order, it seems to be new. Concerning (4), we observe that one of us had already proved it for all $\mu$ belonging to the sequence $\{(1 / 2)+(1 / 2 q)\}(q=1,2,3 . \cdots)$ [Notices Amer. Math. Soc., 1967, Abstract 643-23]. Theorem A remains true in the limiting case $\mu=1$; with the restriction ${ }^{2} \lambda<+\infty$ it follows from a sharpened form due to Edrei and Fuchs [3, Théorème 3, p. 264] of a result of Pfluger [6].

Finally we remark that, if (2) is replaced by $0 \leqq \mu \leqq \frac{1}{2}$, and if $\delta(\infty, f)=1$, then

$$
\Delta(f)=\delta(\infty, f)=1
$$

and $\nu(f)=1$. This follows immediately from an older result of one of us $[1$, Theorem 3, p. 4$]$.

1. Auxiliary notions and notations. Our proof depends essentially on the following fact. If equality holds in (3), there must exist infinitely many, well chosen intervals

$$
R_{m}^{\prime} \leqq r \leqq R_{m}^{\prime \prime} \quad(m=1,2,3, \cdots),
$$

such that

$$
\lim _{m \rightarrow \infty} R_{m}^{\prime}=+\infty, \quad \lim _{m \rightarrow \infty} \frac{R_{m}^{\prime \prime}}{R_{m}^{\prime}}=+\infty,
$$

and such that, if $r$ and $t$ lie in the intervals (1.1), then $T(t, f) / T(r, f)$ is very close to $(t / r)^{\mu}$.

A precise formulation requires a few definitions and notations:

I. Pólya peaks of order $\mu$. $A$ positive sequence $r_{1}, r_{2}, r_{3}, \cdots$ of numbers tending to $+\infty$ is said to be a sequence of Polya peaks, of order $\mu$ of $T(r)$, if it is possible to find three positive sequences $\left\{r_{m}^{\prime}\right\}$, $\left\{r_{m}^{\prime \prime}\right\},\left\{\epsilon_{m}\right\}$, such that, as $m \rightarrow+\infty$,

$$
r_{m}^{\prime} \rightarrow+\infty, \quad\left(r_{m} / r_{m}^{\prime}\right) \rightarrow+\infty, \quad\left(r_{m}^{\prime \prime} / r_{m}\right) \rightarrow+\infty, \quad \epsilon_{m} \rightarrow 0,
$$

and such that the inequalities

$$
r_{m}^{\prime} \leqq t \leqq r_{m}^{\prime \prime} \quad\left(m>m_{0}\right),
$$

${ }^{2}$ Some of the arguments used in our proof of Theorem 1 would make it possible to omit this restriction. 
imply

$$
T(t) / T\left(r_{m}\right) \leqq\left(1+\epsilon_{m}\right)\left(t / r_{m}\right)^{\mu} .
$$

We take for granted the fact that, if $f(z)$ is of lower order $\mu$, then $T(r)=T(r, f)$ has a sequence of Pólya peaks of order $\mu$. A proof will be found in [2, pp. 85-86].

II. QUANTITIES $u(f)$ AND $v(f)$ DEFINED IN TERMS OF EXCEPTIONAL SETS. Let $\varepsilon$ denote a measurable subset of the axis $r>0$ and let $\varepsilon\left[r^{\prime}, r^{\prime \prime}\right]$ be the portion of $\varepsilon$ which lies in the interval $\left[r^{\prime}, r^{\prime \prime}\right]$. We say that $\varepsilon$ has density zero if

$$
\lim _{r \rightarrow \infty} \frac{\text { meas } E[0, r]}{r}=0 .
$$

We consider systematically the two quantities

$$
\lim _{r \rightarrow \infty ; r \notin \varepsilon} \frac{N(r, 1 / f)}{T(r)}=u(f)=u, \quad \lim _{r \rightarrow \infty ; r \notin \varepsilon} \frac{N(r, f)}{T(r)}=v(f)=v,
$$

as well as the analogous quantities $u\left(f^{\prime}\right)$ and $v\left(f^{\prime}\right)$.

The set $\varepsilon$, which is avoided as $r \rightarrow+\infty$, is always assumed to be of density zero.

If $\mathcal{E}$ is a bounded set, the formulae (1.2) reduce to

$$
u=1-\delta(0, f), \quad v=1-\delta(\infty, f) .
$$

III. DEFINITIONS OF THE SECTOR $S$ AND OF THE COUNTING FUNCTION $n(\mathcal{S}, f)$. The sector

$$
\S=\S\left(\omega, \gamma ; R^{\prime}, R^{\prime \prime}\right)
$$

is defined to be the set of all points $z$ satisfying the inequalities

$$
\omega-\gamma \leqq \arg z \leqq \omega+\gamma \quad(0<\gamma<\pi), \quad R^{\prime} \leqq|z| \leqq R^{\prime \prime} .
$$

We extend in an obvious way Nevanlinna's notation and denote by $n(\delta, f)$ the number of poles of $f(z)$ which fall in the sector $\mathcal{S}$.

\section{Statement of the main result.}

THEOREM 1. Let $f(z)$ be a meromorphic function of lower order $\mu(0<\mu<1)$ and let $u$ and $v$ be defined by (1.2).

I. Then

$$
\sin ^{2} \pi \mu \leqq u^{2}+v^{2}-2 u v \cos \pi \mu \text {. }
$$

Moreover, $v \leqq \cos \pi \mu$ implies $u=1$ and $u \leqq \cos \pi \mu$ implies $v=1$. 
II. Let $\left\{r_{m}\right\}$ be a sequence of Polya peaks of order $\mu$ of $T(r)$ and let $E_{\infty}(r)$ and $E_{0}(r)$ be sets of $\theta(-\pi \leqq \theta<\pi)$ defined by

$$
E_{\infty}(r)=\left\{\theta:\left|f\left(r e^{i \theta}\right)\right| \geqq r^{\alpha}\right\}, \quad E_{0}(r)=\left\{\theta:\left|f\left(r e^{i \theta}\right)\right| \leqq r^{-\alpha}\right\},
$$

where $\alpha$ is an arbitrary, nonnegative constant.

Assume that equality holds in (2.1) and that $u<1, v<1$. Then all the following limits exist and satisfy the relations stated

$$
\begin{aligned}
\lim _{m \rightarrow \infty} \text { meas } E_{\infty}\left(r_{m}\right)=s(\infty)=\frac{2}{\mu} \cos ^{-1} v & \left(0<\cos ^{-1} v \leqq \frac{\pi}{2}\right), \\
\lim _{m \rightarrow \infty} \text { meas } E_{0}\left(r_{m}\right)=s(0)=\frac{2}{\mu} \cos ^{-1} u & \left(0<\cos ^{-1} u \leqq \frac{\pi}{2}\right), \\
s(0)+s(\infty)=2 \pi . &
\end{aligned}
$$

Moreover, there exist three positive sequences $\left\{R_{m}^{\prime}\right\},\left\{R_{m}^{\prime \prime}\right\},\left\{\tilde{\epsilon}_{m}\right\}$ such that, as $m \rightarrow+\infty$,

$$
R_{m}^{\prime} \rightarrow+\infty, \quad r_{m} / R_{m}^{\prime} \rightarrow+\infty, \quad R_{m}^{\prime \prime} / r_{m} \rightarrow+\infty, \quad \tilde{\epsilon}_{m} \rightarrow 0,
$$

and such that

$$
R_{m}^{\prime} \leqq t \leqq R_{m}^{\prime \prime} \quad\left(m>m_{0}\right)
$$

imply

$$
\left(t / r_{m}\right)^{\mu}\left(1+\tilde{\epsilon}_{m}\right)^{-1} \leqq T(t) / T\left(r_{m}\right) \leqq\left(t / r_{m}\right)^{\mu}\left(1+\tilde{\epsilon}_{m}\right)
$$

and

(2.3) $\mu u-\tilde{\epsilon}_{m} \leqq n(t, 1 / f) / T(t) \leqq \mu u+\tilde{\epsilon}_{m}, \quad \mu v-\tilde{\epsilon}_{m} \leqq n(t, f) / T(t) \leqq \mu v+\tilde{\epsilon}_{m}$.

There also exist a real sequence $\left\{\omega_{m}\right\}$ and a positive sequence $\left\{\eta_{m}\right\}$ such that, as $m \rightarrow+\infty, \eta_{m} \rightarrow 0$ and

$$
\begin{aligned}
& n\left(\$\left(\omega_{m}, \eta_{m} ; R_{m}^{\prime}, R_{m}^{\prime \prime}\right), 1 / f\right)=n\left(R_{m}^{\prime \prime}, 1 / f\right)+o\left(T\left(r_{m}\right)\right), \\
& n\left(S\left(\omega_{m}+\pi, \eta_{m} ; R_{m}^{\prime}, R_{m}^{\prime \prime}\right), f\right)=n\left(R_{m}^{\prime \prime}, f\right)+o\left(T\left(r_{m}\right)\right) .
\end{aligned}
$$

Assertion II of the above theorem is closely related to a tauberian theorem of Edrei and Fuchs [5, Theorem 1, p. 340]. The inequalities (2.2) and (2.3) are "local." Their validity is confined to the intervals $\left[R_{m}^{\prime}, R_{m}^{\prime \prime}\right]$ and examples show that the inequalities are no longer true for unrestricted values of $t$.

The relations (2.3) and (2.4) determine, in the annulus $R_{m}^{\prime} \leqq|z|$ $\leqq R_{m}^{\prime \prime}$, the moduli and the arguments of the zeros and poles of $f(z)$ 
with such precision that an asymptotic evaluation of $f(z)$ becomes possible on suitable circumferences.

The steps which lead to the proof of assertion (4) of Theorem A may be described as follows.

1. The simultaneous consideration of $f(z)$ and $f^{\prime}(z)$ shows that $\Delta(f)=2-\sin \pi \mu\left(\frac{1}{2}<\mu<1\right)$ and $\delta(\infty, f)=1$ imply

$$
u\left(f^{\prime}\right)=\sin \pi \mu, \quad v\left(f^{\prime}\right)=0 .
$$

2. The relations (2.5) make it possible to apply assertion II of Theorem 1 to $f^{\prime}(z)$ and hence to obtain the asymptotic evaluation of $f^{\prime}(z)$ on suitable circumferences. This shows that, on single arcs of these circumferences, $f^{\prime}$ is so small that $f$ is practically constant. On the complementary arcs $f$ is very large. It is easily shown that this behavior limits to two the number of deficient values of $f(z)$.

\section{REFERENCES}

1. A. Edrei, The deficiencies of meromorphic functions of finite lower order, Duke Math. J. 31 (1964), 1-22.

2. - Sums of deficiencies of meromorphic functions, J. Analyse Math. 14 (1965), 79-107.

3. A. Edrei and W. H. J. Fuchs, Valeurs deficientes et valeurs asymptotiques des fonctions meromorphes, Comment. Math. Helv. 33 (1959), 258-295.

4. - The deficiencies of meromorphic functions of order less than one, Duke Math. J. 27 (1960), 233-250.

5. - Tauberian theorems for a class of meromorphic functions with negative zeros and positive poles, Proc. Int. Conf. in Function Theory, Erevan (1965), 339-358.

6. A. Pfluger, Zur Defektrelation ganzer Funktionen endlicher Ordnung, Comment. Math. Helv. 19 (1946/47), 91-104.

SYRACUSE UNIVERSITY 